\title{
Nucleation- and Emergence-Limited Growth of Ice from Pores
}

\author{
James M. Campbell and Hugo K. Christenson \\ School of Physics and Astronomy, University of Leeds, Woodhouse Lane, Leeds LS2 9JT, United Kingdom
}

(Received 18 October 2017; revised manuscript received 21 February 2018; published 16 April 2018)

\begin{abstract}
Nucleation of ice from vapor on atmospheric aerosols has been attributed to the condensation and freezing of supercooled water in small pores. Here we use wedge pores on mica to directly observe the growth of ice in confinement prior to the growth of bulk crystals. We report a transition in behavior with a decreasing temperature: At low temperatures, the limiting step is not nucleation but a free energy barrier associated with the growth of ice through a narrow pore mouth to become a bulk phase.
\end{abstract}

DOI: 10.1103/PhysRevLett.120.165701

Ice does not nucleate homogeneously in the atmosphere. It is dependent upon atmospheric aerosols (small fragments of mineral dust, soot, and biological entities among others) on which to form a heterogeneous nucleus. Some aerosol species are seen to be much more active than others [1,2]. Much of this variation is certainly due to differences in surface chemistry, but there has been considerable recent interest in how surface topography may also play an important role $[3,4]$. Many experimental nucleation studies have shown topography to be important [5-9], especially in the deposition mode, i.e., nucleation with no visible liquid phase beforehand. Deposition mode nucleation is traditionally ascribed to the direct nucleation of ice from vapor [10-13]; however, an alternative explanation-first proposed by Fukuta [14] and since termed the pore condensation and freezing model — has recently attracted interest $[15,16]$. It is suggested that small pores in a surface fill with supercooled water through capillary condensation, which then freezes; this has been experimentally verified for organic substances by Christenson and co-workers $[17,18]$. A review by Marcolli looked at literature results on deposition mode ice nucleation and concluded that pore condensation and freezing offered the best explanation [19]. However, until now it has been impossible to make direct observations of processes occurring in confined geometries which precede the growth of a bulk ice crystal.

Previously, we have made such observations for several different organic crystals growing from vapor [20]. Using a type of feature found on surfaces of cleaved Muscovite mica known as "pockets" which make ideal model pores, we were able to observe solid condensates within the pockets prior to the appearance of bulk crystals. These bulk crystals started to grow only after a threshold supersaturation had been achieved. A limited number of ice nucleation experiments were performed at temperatures down to $-45^{\circ} \mathrm{C}$, and, although the pattern of growth was suggestive of growth via a confined phase, none was observed prior to the growth of bulk crystals.
In this Letter, we present the successful observation of the growth of ice in confinement preceding - and leading to - the growth of bulk ice crystals. Furthermore, we report a new phenomenon not previously considered within the pore condensation and freezing literature: a transition from nucleation-limited growth of crystals at higher temperatures to growth limited by an escape from confinement through a narrow pore mouth at lower temperatures.

We again use mica pockets as convenient model pores. They are cavities between two mica layers along a step edge, featuring a highly acute wedge geometry coming to an angle below $1^{\circ}$ along the $U$-shaped apex. In reflected light, they are visible as a series of interference fringes between the two closely spaced mica surfaces. Material condensed in the wedge apex is easily visible if it grows to have an interface height above about $30 \mathrm{~nm}$. Figure 1

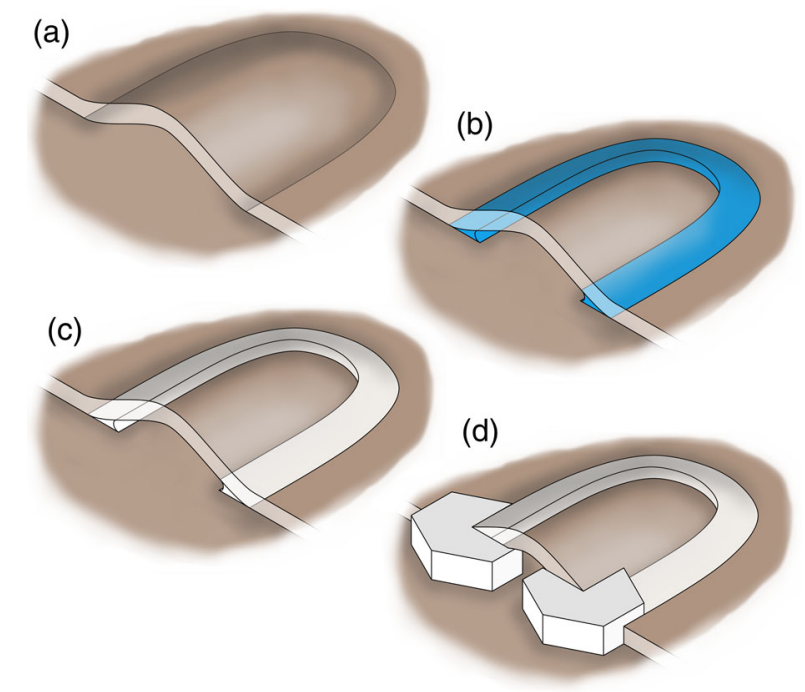

FIG. 1. Illustration of ice growth from a mica pocket: (a) an empty pocket; (b) a capillary condensate of supercooled water forms within the pocket; (c) the water freezes to produce a solid condensate; (d) bulk ice crystals emerge from the pocket corners. 


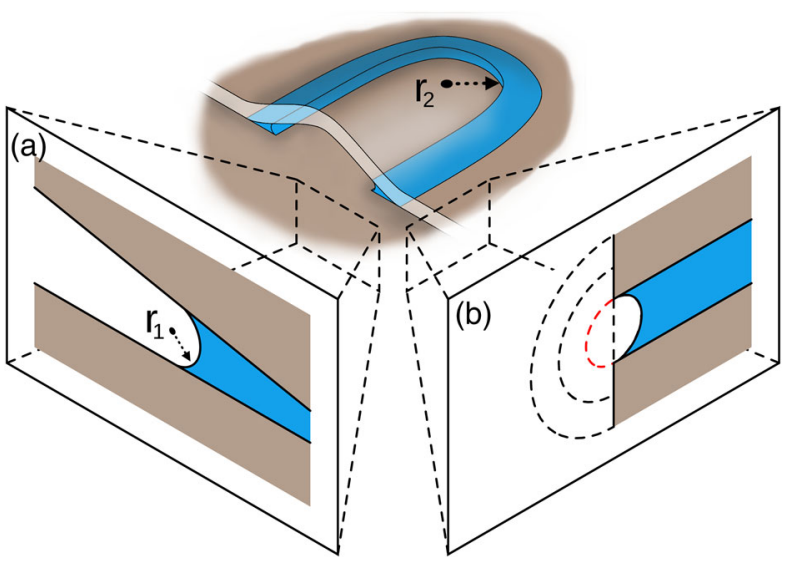

FIG. 2. Two schematic cross sections through a liquid condensate in perpendicular planes: (a) a condensate with radius of curvature $r_{1}$ grows within wedge geometry as the saturation increases; (b) after the condensate freezes, the dashed lines show the evolution the ice interface would have to follow to emerge into a bulk phase (here assuming $\theta^{i} \approx 90^{\circ}$ ). The red line shows the position of maximum convex curvature.

illustrates the expected sequence of pore condensation and freezing in a mica pocket. Liquid water first condenses in the apex; this then freezes, and finally bulk ice crystals may grow from the two pocket "corners" (the points where the wedge apex meets the step edge), growing much faster than the confined ice due to a lower diffusion barrier.

A liquid condensate should be able to form in conditions undersaturated with respect to bulk water, because the geometry allows it to form a tightly concave interface. The vapor pressure $p_{c}$ over a surface with radius of curvature $r$ relates to that over a flat surface $p_{o}$ by the Kelvin equation

$$
\ln \frac{p_{c}}{p_{o}}=\frac{\gamma V_{m}}{r R T}
$$

where $\gamma$ is the surface tension, $V_{m}$ the molar volume, $R$ the gas constant, and $T$ the temperature. $r$ (defined as negative for a concave interface) is a function of two radii of curvature $r_{1}$ and $r_{2}$ (shown in Fig. 2), such that $r^{-1}=$ $r_{1}^{-1}+r_{2}^{-1}$. However, we assume that $r_{2}^{-1}$ is negligible, such that $r \approx r_{1}$. Provided the phase has a contact angle $\theta$ below $90^{\circ}$, the interface will be concave in an acute wedge, and a condensate (either solid or liquid) may exist in undersaturated conditions, saturation $S$ defined here as

$$
S=\frac{p}{p_{o}}
$$

where $p$ is the ambient partial pressure. The equilibrium condensate size increases towards infinity as $S$ increases towards unity.

Mica substrates were prepared by cleaving mica in a laminar flow cabinet until an area was found featuring only a single pocket defect of suitable size (the median pocket size was $1.1 \mathrm{~mm}$ around the wedge apex). Substrates were back-painted in black enamel to improve optical properties before being attached to a copper plate within the experimental cell using silicone-free thermal grease. The temperature was controlled using a liquid nitrogen chiller and measured using a resistance thermometer. The gas humidity was controlled using two mass flow controllers to mix dry nitrogen with nitrogen bubbled through a column of water. The total gas flow was kept at $1.25 \mathrm{~L} \mathrm{~min}^{-1}$ with the humidity varied by adjusting the flow rate through the water column and the temperature of the water. The gas flowed for at least $90 \mathrm{~min}$ before the start of each experiment to allow the system to reach equilibrium.

Experiments were performed in conditions of decreasing temperature and increasing saturation at a fixed frost point, this being the temperature at which ice would be in equilibrium with the vapor. In each experiment, the substrate temperature was reduced at $0.2^{\circ} \mathrm{C} \mathrm{min}^{-1}$ from a temperature at least $1{ }^{\circ} \mathrm{C}$ above the frost point, and periodic photographs taken using an optical microscope with greenfiltered LED illumination. After bulk crystals were observed, the frost point of the gas was found by taking the substrate temperature back and forth across the frost point at $0.05^{\circ} \mathrm{C} \mathrm{min}^{-1}$. The frost point could be identified as the temperature at which the crystals neither grew nor shrank. Substrates were placed with the pocket directly over the coldest point in the cell, making vapor depletion from crystals nucleated out of shot unlikely. The absolute error in the temperature is $\pm 0.4^{\circ} \mathrm{C}$, with a relative error of $\pm 0.2^{\circ} \mathrm{C}$ between runs. The error in saturation is \pm 0.02 .

Figure 3 shows two examples of ice growth from a mica pocket. The pattern of growth-with bulk crystals at the corners and more limited fingering growth along the wedge apex-is exactly that expected for crystallization from a supercooled liquid capillary condensate, as shown schematically in Fig. 1. In many cases, as in Figs. 3(d)-3(f), ice is clearly seen growing within the pocket significantly before bulk crystals begin to grow from the corners.

The style of growth was dependent on the frost point at which the experiment was performed. At lower frost points (below about $-42{ }^{\circ} \mathrm{C}$ ), condensates were observed before bulk crystals on most runs, and typically either only one bulk crystal grows, or crystals emerge from the two corners at two distinct times. At higher frost points (between -42 and $-35^{\circ} \mathrm{C}$ ), the two crystals usually appear simultaneously, with no visible condensate beforehand, although ice growth was seen along the wedge apex subsequent to the appearance of bulk crystals. At frost points above $-35^{\circ} \mathrm{C}$, no crystallization is observed within the pocket, and ice forms by condensation and freezing on the flat mica surface. Drops of liquid water condensed across the whole mica surface, some of which then froze.

This trend is demonstrated in Fig. 4, which plots the temperature and saturation with respect to ice $S^{i}$ (the superscript $w$ represents properties of water and $i$ those 

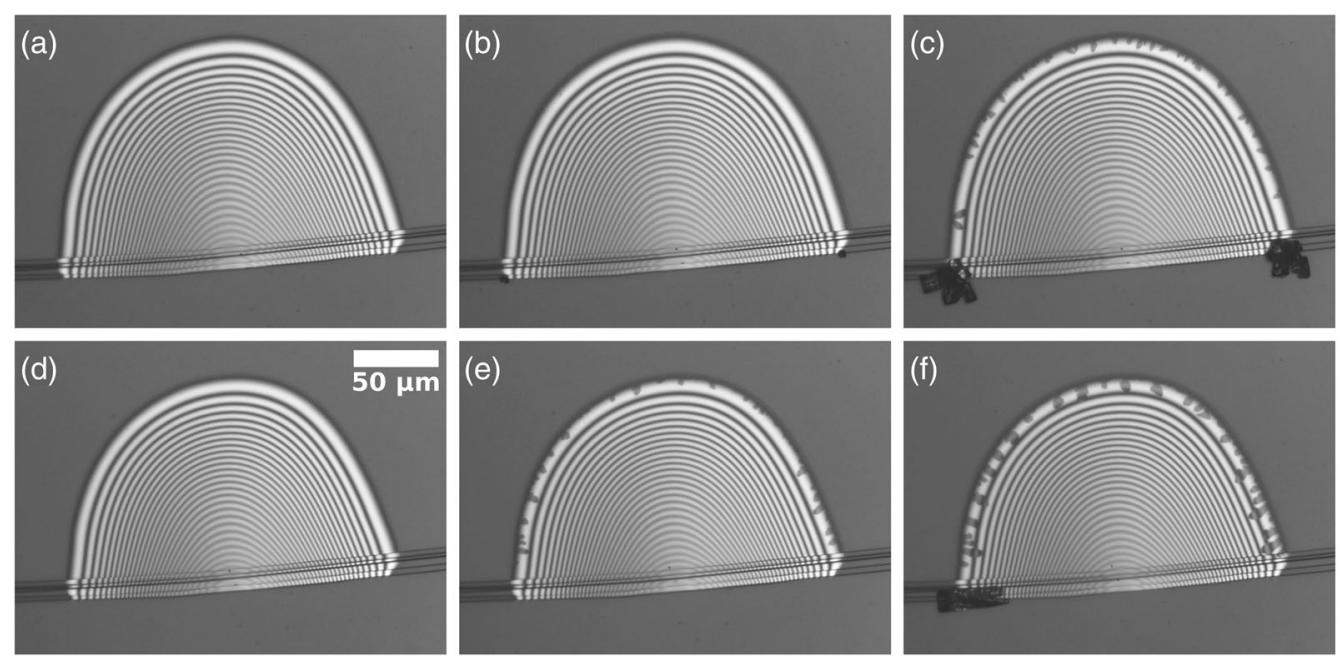

FIG. 3. Two representative time sequences of growth in the same pocket: at frost points $-37.4{ }^{\circ} \mathrm{C}$ (a) $-(\mathrm{c})$ and $-48.5^{\circ} \mathrm{C}(\mathrm{d})-(\mathrm{f})$. For the upper sequence, two bulk crystals emerge simultaneously (b) before visible growth along the wedge apex (c). For the lower sequence, a confined solid phase is visible (e) before the emergence of a single bulk crystal (f).

of ice) at the moment of the appearance of a bulk crystal for every experiment. Runs with a simultaneous appearance of both bulk crystals are colored in red, and condensates visible prior to bulk crystals are indicated by a tail. It may be easily seen that the runs with simultaneous bulk crystals are strongly clustered in conditions of high temperature and high saturation, while those with visible condensates beforehand are mostly found in the lower-temperature conditions. With only a single exception, runs with simultaneous growth of bulk crystals do not feature visible condensates prior to those crystals' appearance.

Let us first assume a simple view of the process: that water condenses into the acute apex of mica pockets and then freezes, to form a bulk crystal with no other impediment. What would we expect to see in this case? As the pocket apex is a single uninterrupted wedge, we would expect a single uninterrupted condensate all along it, from corner to corner. This would be too small to observe at typical saturations [20], but, assuming nucleation occurs in conditions $S^{i}>1$, there would follow the immediate growth of bulk crystals from both corners and along the wedge apex. This agrees with what we observe at higher temperatures but not at lower ones.

At lower temperatures, there is clearly ice growing within the pocket substantially prior to growth outside of it. As the initial condensate is still assumed to be a continuous one, we must introduce a new impediment to growth to explain why bulk crystals are not appearing in supersaturated conditions. For this, we introduce the concept of a free energy barrier for a phase to grow out of a narrow gap at low supersaturation, a process from here on called "emergence." This "emergence barrier," which we have discussed before in reference to the nucleation of organic compounds [20] and has been observed in a simulation [21,22], is illustrated in Fig. 2(b). If a phase has $\theta$ above $0^{\circ}$, then it must form a convex interface to grow into a bulk phase, passing through a maximum curvature. From Eq. (1), this maximum corresponds to a threshold supersaturation below which an emergence barrier exists. The narrower the pore mouth, the higher the threshold.

Taking emergence into account, we can expect that a process may be either nucleation-limited or emergencelimited. Nucleation will be the limiting step if, at the moment the condensate freezes, $S^{i}$ is sufficient to allow immediate emergence without a barrier. Emergence will be the limiting step if the condensate freezes when $S^{i}$ is low enough for a barrier to exist. Instead, it will grow within the confines of the pocket until emergence is possible. There is no reason to expect emergence at both corners simultaneously (discussed later), and when one emerges slightly before the other it will suppress the other's growth through vapor depletion. Therefore, the simultaneous appearance of two bulk crystals is not expected.

We can predict the saturation at which an emergence barrier ceases to exist. If we assume that $\theta^{w} \approx 0^{\circ}$ (we have measured the contact angle of water on mica in the range $2^{\circ}-3^{\circ}$ ) and that $\theta^{i} \approx 90^{\circ}\left(\theta^{i}\right.$ is unknown, but mica's ineffectiveness as a heterogeneous nucleant of ice [23] implies that $\theta^{i}$ is significantly greater than $\theta^{w} ; \theta^{i} \geq 90^{\circ}$ gives a maximum emergence barrier), and we assume the simplest geometry shown in Fig. 2(b), we reach the condition $r^{*} \approx-r$. Thus, using Eq. (1),

$$
\frac{\ln S^{i}}{\ln S^{w}} \approx-\frac{\gamma^{i} V_{m}^{i}}{\gamma^{w} V_{m}^{w}}
$$

which is displayed as the turquoise line in Fig. 4. This should represent the lower boundary of the nucleationlimited region, as above this line any condensate which freezes should be able to immediately emerge. This is 


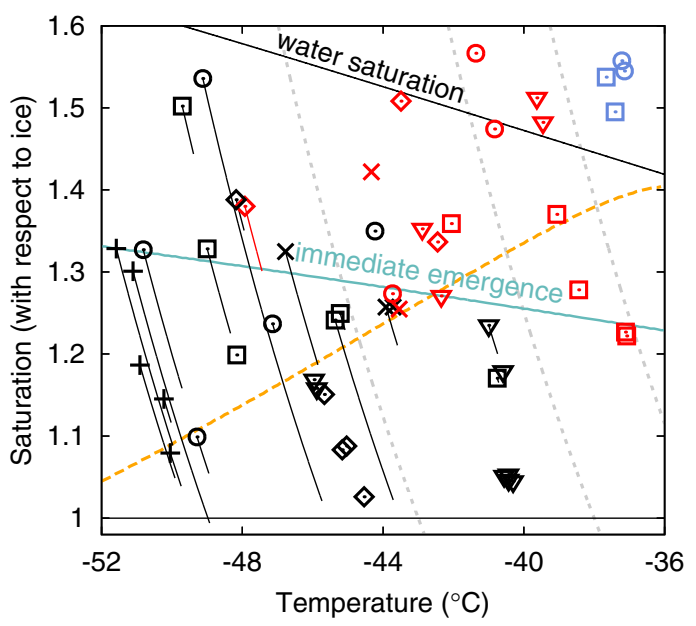

FIG. 4. (a) Temperature and saturation at the first emergence of a bulk crystal. Red symbols represent the simultaneous growth of bulk crystals from both pocket corners, black symbols represent a bulk crystal at a single corner, and blue symbols represent condensation and freezing outside the pocket. The six different symbols represent six unique mica pockets. Tails on symbols are lines of constant frost point, representing periods where a condensate was visible within a pocket prior to emergence. The turquoise line is from Eq. (3), above which a liquid condensate may immediately emerge upon freezing. The orange dashed line is an approximate prediction of the nucleation point in a typical pocket. The gray dotted lines represent frost points $-43^{\circ},-38^{\circ}$, and $-35^{\circ} \mathrm{C}$.

supported by the data; the region of red points is seen to be exclusively close to or above the line, while the region of black points is seen close to or below the line or (with one exception) having a tail extending to below or close to the line, indicating that the condensate froze before the line was reached. Note that the nucleation of the condensate would have occurred an unknown time before the first visible condensate shown in the figure, depending on the saturation, magnification, growth morphology, and quality of focus. For this same reason, it cannot be assumed that the absence of an observable condensate is evidence of the absence of confined ice before bulk emergence.

A temperature dependence between nucleation- or emergence-limited growth follows naturally as a consequence that, at lower temperatures, condensates are more likely to freeze at lower saturation. We have predicted the typical saturation at which we expect nucleation in an average-sized pocket. This is detailed in Supplemental Material [24], but to summarize, we find the volume of a condensate in equilibrium with the vapor and assume homogeneous nucleation, using an empirical form for the volumetric nucleation rate in an aqueous solution from Koop et al. [25]. The model also assumes the dissolution of potassium carbonate from the surface, which is expected to form from unbound potassium freed by cleavage [26], meaning that small condensates are likely to be highly concentrated. The predicted nucleation curve agrees well with the observed transition from nucleation-limited to emergence-limited growth. It is, however, worth remembering that predictions of nucleation rates at such low temperatures are highly approximate and that in any case the scatter of data is so great that the nucleation process clearly cannot be described so simply. We suspect that some pockets may contain contaminant nucleants and that the process of performing repeat experiments may lead to the local concentration of soluble material well above (or below) that predicted by a homogeneous distribution across the surface. Nevertheless, the nucleation calculation acts as a rough check that the transition is close to the expected temperature. This transition is not expected to be a sharp one; even in a perfect system, there would be conditions in which growth may be either nucleation- or emergencelimited based on stochastic variation. For our data, between frost points $-35^{\circ}$ and $-38^{\circ} \mathrm{C}$ we see purely nucleationlimited growth, below $-43^{\circ} \mathrm{C}$ we see purely emergencelimited growth, and between $-38^{\circ}$ and $-43^{\circ} \mathrm{C}$ there is a mix of both (see Fig. 4).

While the variability of nucleation can explain the scatter of saturations in the nucleation-limited regime, other processes contribute to the scatter in the emergence-limited regime. As seen in Fig. 4, the length of time between a condensate becoming visible and the emergence of a bulk crystal is highly variable between experiments and even between different runs within the same pocket in the same conditions. Unlike a liquid condensate, a growing ice condensate does not form a smooth interface but an array of needles (see Fig. 3), as growth is limited by the diffusion of material into the acute geometry, and there is a lower diffusion barrier to the tips of the needles than to their base. This makes it difficult to predict the local interface height close to the corners; it also makes it difficult to predict the local saturation, as the rapidly growing ice is likely to deplete water vapor from its vicinity. It can be imagined that a condensate which produces a growing needle at or very close to the corner will very quickly emerge into a bulk crystal, while one which produces a needle a little set back from the corner will deplete vapor from the corner and emergence will take a lot longer. A more detailed discussion, with photographs of some illustrative examples, is presented in Supplemental Material [24].

In conclusion, we have directly observed ice crystal growth via a confined phase within a wedge pore, as predicted by the pore condensation and freezing model. Also, by observing ice growing in confinement in supersaturated conditions without immediately leading to the growth of a bulk crystal, we have demonstrated that nucleation is not always the only process limiting ice formation from pores. There is a transition with a decreasing temperature from nucleation-limited growth to growth limited by emergence through a narrow pore mouth. The implication is that an effective nucleation site needs to have not only the geometry and chemistry to 
promote condensation and freezing of water, but also a geometry suitable for bulk emergence at ambient saturation. It is possible that the criteria for an effective nucleation site are temperature dependent, depending on whether ice is growing in the nucleation-limited or emergence-limited regime. In this study, we have studied only a very particular geometry and substrate (pockets on mica), but there is nothing system-specific about our results. The same principles should apply for different pore geometries and different materials, including for real atmospheric aerosols inducing ice nucleation in the atmosphere.

H. K. C. acknowledges a grant from the Leverhulme Trust (RPG-2014-306), and we thank F. C. Meldrum for useful comments.

[1] C. Hoose and O. Möhler, Atmos. Chem. Phys. 12, 9817 (2012).

[2] J. D. Atkinson, B. J. Murray, M. T. Woodhouse, T. F. Whale, K. J. Baustian, K. S. Carslaw, S. Dobbie, D. O'Sullivan, and T. L. Malkin, Nature (London) 498, 355 (2013).

[3] N. Hiranuma, N. Hoffmann, A. Kiselev, A. Dreyer, K. Zhang, G. Kulkarni, T. Koop, and O. Möhler, Atmos. Chem. Phys. 14, 2315 (2014).

[4] Y. Bi, B. Cao, and T. Li, Nat. Commun. 8, 15372 (2017).

[5] M. Järn, S. Areva, V. Pore, J. Peltonen, and M. Linden, Langmuir 22, 8209 (2006).

[6] Y. Diao, T. Harada, A. S. Myerson, T. A. Hatton, and B. L. Trout, Nat. Mater. 10, 867 (2011).

[7] Y. Diao, A. S. Myerson, T. A. Hatton, and B. L. Trout, Langmuir 27, 5324 (2011).

[8] A. Sengupta Ghatak and A. Ghatak, Langmuir 29, 4373 (2013).
[9] C. W. Gurganus, J. C. Charnawskas, A. B. Kostinski, and R. A. Shaw, Phys. Rev. Lett. 113, 235701 (2014).

[10] J. G. Buijnsters, L. Vázquez, and J. J. ter Meulen, Diam. Relat. Mater. 18, 1239 (2009).

[11] A. Portavoce, M. Kammler, R. Hull, M. C. Reuter, and F. M. Ross, Nanotechnology 17, 4451 (2006).

[12] J. L. Holbrough, J. M. Campbell, F. C. Meldrum, and H. K. Christenson, Cryst. Growth Des. 12, 750 (2012).

[13] J. M. Campbell, F. C. Meldrum, and H. K. Christenson, Cryst. Growth Des. 13, 1915 (2013).

[14] N. Fukuta, J. Atmos. Sci. 23, 741 (1966).

[15] R. Wagner, A. Kiselev, O. Möhler, H. Saathoff, and I. Steinke, Atmos. Chem. Phys. 16, 2025 (2016).

[16] R. O. David, F. Mahrt, C. Marcolli, J. Fahrni, D. Brühwiler, U. Lohmann, and Z. A. Kanji, Geophys. Res. Abstr. 19, 16293 (2017)

[17] T. Kovács, F. C. Meldrum, and H. K. Christenson, J. Phys. Chem. Lett. 3, 1602 (2012).

[18] H. K. Christenson, CrystEngComm 15, 2030 (2013).

[19] C. Marcolli, Atmos. Chem. Phys. 14, 2071 (2014).

[20] J. M. Campbell, F. C. Meldrum, and H. K. Christenson, Proc. Natl. Acad. Sci. U.S.A. 114, 810 (2017).

[21] A. J. Page and R. P. Sear, Phys. Rev. Lett. 97, 065701 (2006).

[22] Y. Liu, Y. Men, and X. Zhang, J. Chem. Phys. 137, 104701 (2012).

[23] J. M. Campbell, F. C. Meldrum, and H. K. Christenson, J. Phys. Chem. C 119, 1164 (2015).

[24] See Supplemental Material at http://link.aps.org/ supplemental/10.1103/PhysRevLett.120.165701 for details of nucleation rate calculation, and a discussion of ice growth dynamics within pockets.

[25] T. Koop, B. Luo, A. Tsias, and P. Thomas, Nature (London) 406, 611 (2000).

[26] H. K. Christenson and N. H. Thomson, Surf. Sci. Rep. 71, 367 (2016). 